\title{
Ulcération linguale et dysphagie à l'origine du diagnostic d'une tuberculose pulmonaire
}

\author{
D’Elbée $\mathrm{JM}^{1}$, Bernard $\mathrm{N}^{2}$, Bouyer $\mathrm{M}^{2}$, Vandenhende $\mathrm{MA}^{2}$, Lacoste $\mathrm{D}^{2}$, Bonnet $\mathrm{F}^{2}$, \\ Michaux $\mathrm{C}^{2}$, Morlat $\mathrm{P}^{2}$, Fricain $\mathrm{JC}^{1}$ \\ ${ }^{1}$ Pôle Odontologie et Santé buccale, CHU, Bordeaux, France \\ ${ }^{2}$ Service de Médecine interne et Maladies infectieuses, Hôpital Saint André, CHU, Bordeaux, \\ France
}

La prévalence de la tuberculose en France est de 9/100 000 (Antoine 2009). Les manifestations buccales au cours de la tuberculose sont rares (incidence $1,4 \%$ ) touchant alors le plus souvent la langue (Fernando et al. 2009). Un nouveau cas d'atteinte linguale se manifestant par une dysphagie à l'origine du diagnostic de tuberculose est rapporté.

Un patient âgé de 48 ans, cuisinier au chômage, ayant comme seul antécédent un tabagisme sevré de 10 PA, a été hospitalisé en avril 2010 pour une altération franche de l'état général avec dysphagie. Il présentait un amaigrissement de $12 \mathrm{~kg}$, une fièvre, des sueurs nocturnes, une toux sèche, des crachats hémoptoïques et une dysphagie persistante. L'examen clinique objectivait une cachexie (IMC à 17,5), une fièvre en plateau à $39^{\circ}$, deux adénopathies submandibulaires gauches et une lésion linguale suspecte et douloureuse. La langue était dépapillée avec une large ulcération siégeant sur le bord droit, recouverte par un enduit blanchâtre en périphérie.

Le bilan biologique montrait des globules blancs à $11,3 \mathrm{G} / \mathrm{L}$ avec $8,66 \mathrm{G} / \mathrm{L}$ de polynucléaires neutrophiles, $1,15 \mathrm{G} / \mathrm{L}$ de lymphocytes, une hémoglobine à $11,8 \mathrm{~g} / 100 \mathrm{ml}$, une CRP à 144 $\mathrm{mg} / \mathrm{L}$, une protidémie à $53 \mathrm{~g} / \mathrm{L}$, un taux de fibrinogène à $10,3 \mathrm{~g} / \mathrm{L}$, une albuminémie à 19,4 $\mathrm{g} / \mathrm{L}$; les sérologies pour le VIH, le VHB et le VHC étaient négatives. La radiographie pulmonaire mettait en évidence une miliaire (opacité micronodulaire), confirmée par le CTscan thoracique.

Devant ce tableau, une quadrithérapie anti-tuberculeuse par voie intraveineuse a été entreprise sans attendre le résultat de l'examen des crachats qui sont revenus positifs pour Mycobacterium tuberculosis. Des biopsies de langue ont été réalisées à J8. L'examen anatomopathologique a mis en évidence un important infiltrat inflammatoire comportant des lympocytes, des plasmocytes, des histiocytes et des cellules épithélioïdes, mais il n'y avait pas de nécrose caséeuse et ni de cellules géantes multinucléées. Après une douzaine de jours, Le patient était apyrétique et il a recommencé à s'alimenter. La lésion linguale était totalement cicatrisée 21 jours après le début du traitement.

Les lésions buccales de tuberculose sont généralement secondaires à une localisation pulmonaire (54\% des cas). L'inoculation de la muqueuse buccale se ferait soit par les crachats (rôle favorisant d'une lésion locale), soit par voie hématogène. Elles se traduisent le plus souvent par une ulcération douloureuse, avec des bords irréguliers, une base légèrement indurée et jaunâtre (Tovarus et al. 2008). Cet aspect peut faire évoquer un carcinome épidermoïde, surtout si l'ulcération survient chez un sujet alcoolo-tabagique. L'examen direct ou la culture ne sont pas toujours positifs (ils sont positifs dans un peu plus de $50 \%$ des cas) et la biopsie n'est pas toujours contributive. C'est souvent l'évolution de la lésion sous traitement qui permet de confirmer le diagnostic.

Le diagnostic de tuberculose linguale doit être évoqué lorsqu'il existe une ulcération linguale dans un contexte de tuberculose déjà connue, en particulier pulmonaire. Lorsque l'ulcération est cliniquement isolée, le diagnostic de carcinome épidermoïde est le plus souvent évoqué. La recherche parallèle d'une tuberculose pulmonaire asymptomatique par radiographie pulmonaire est toutefois justifiée si la biopsie linguale ne confirme pas le diagnostic de carcinome épidermoïde (Furugen et al. 2009).

Correspondance : jmtruite@hotmail.fr

This is an Open Access article distributed under the terms of the Creative Commons Attribution-Noncommercial License 3.0, which permits unrestricted use, distribution, and reproduction in any noncommercial medium, provided the original work is properly cited. 